\section{Cationic Lipid-Based Systemic Plasmid Delivery for the Treatment of Angiogenesis-Dependent Tumors}

\author{
Khursheed Anwer, ${ }^{*}$ Clare Meaney, Grace Kao, Nasir \\ Hussain, Robert Shelvin, Rosa M. Earls, Pat Leonard, \\ Alain P. Rolland \& Sean M. Sullivan
}

Valentis, Inc., 8301 New Trails Drive, The Woodlands, Texas 77381-4248

A cationic lipid-based delivery system composed of N [(1-(2,3dioleyloxy)propyl)]-N,N,N-trimethylammonium chloride (DOTMA) and cholesterol, at a 4:1 mole ratio, was developed to express anti-angiogenic gene products from normal and tumor vasculature upon intravenous administration. Plasmid biodistribution, expression, and efficacy were characterized in a syngeneic mouse tumor model using SCCVII (squamous cell carcinoma) cells. Transfection was demonstrated in both lung and primary tumors. Fluorescence microscopy showed that the plasmid-lipid complexes were passively targeted to the tumor vasculature and that the plasmid was localized to endothelial cells. Transgene expression levels were higher in larger tumors, correlating well with increased tumor vasculature as a function of tumor size. The levels of gene expression in subcutaneously implanted tumors peaked 8 to 18 hours after DNA administration, and expression was detectable up to 10 days after a single injection. A second administration, three days following the first, extended the duration of expression in the tumor, whereas no impact on expression was observed in the lungs. IL-12 plasmid produced protein levels in the lung, blood and tumor upon i.v. administration. A doseresponse curve showed that IL-12 could be detected in the tumor with as little as $15 \mathrm{mg}$ of plasmid. This dose, administered three times over a 14 day period, produced a significant inhibition of primary tumor growth. Co-expression of murine endostatin and murine IL-12 produced a synergistic inhibition of tumor growth. These results demonstrate the feasibility of systemically administered plasmid-based gene delivery systems for the treatment of angiogenesis-dependent tumors.

\section{Gene Therapy Accelerates Chronic Duodenal Ulcer Healing in Rats Using VEGF and PDGF Naked DNA or Adenoviral Plasmids}

\author{
X.M. Deng, S. Szabo, M.R. Jadus, T. Khomenko, M. \\ Yoshida, ${ }^{*} M$. Herlyn, ${ }^{*} M$. Nesbit \& W.H. Florsheim
}

Departments of Pathology \& Pharmacology, University of California, Irvine, and Pathology \& Laboratory Medicine Service, VA Medical Center, Long Beach, CA 90822

*Laboratory 484, The Wistar Institute, Philadelphia, PA 19104

Gene therapy has been investigated and used for the treatment for malignant tumors and ischemic diseases in both animal models and clinical settings. Previously, effective ulcer healing with intragastric administration of growth factors such as VEGF (vascular endothelial growth factor) and PDGF (platelet-derived growth factor) was first reported by our group. In this present study, we compared the effects of naked DNA (ND) and adenovirus (AV) transfection of VEGF and PDGF to treat chronic duodenal ulcer induced by the ulcerogen cysteamine in rats. Groups of Sprague-Dawley female rats (180-210 g) were given cysteamine-HCl $(25 \mathrm{mg} / 100 \mathrm{~g}$ by gavage $\mathrm{x} 3$ with $4 \mathrm{hr}$ intervals) to cause duodenal ulcers. Laparotomy on the 3rd day was performed to evaluate ulcer formation and rats with equal severity of duodenal ulcers were randomly divided into 10 groups which received either intraduodenal (i.d.) injection of $0.1 \mathrm{ml} / \mathrm{rat}$ of Tris-EDTA buffer in control group $1(\mathrm{G} 1)$ or $5 \times 10^{8} \mathrm{pfu} / \mathrm{rat}$ of adenovirus in control group 2 (G2), $1 \mathrm{~g} /$ rat (G3) or $100 \mathrm{~g} /$ rat (G4) of naked DNA of VEGF and $5 \times 10^{7} \mathrm{pfu} / \mathrm{rat}$ of PDGF (G5) or $5 \times 10^{8} \mathrm{pfu} / \mathrm{rat}$ of adenovirus vector of PDGF (G6) or VEGF (G7), respectively, and or intravenous (i.v.) injection of $200 \mathrm{~g} / \mathrm{rat}$ of ND of VEGF (G8) and $5 \times 10^{8} \mathrm{pfu} / \mathrm{rat}$ of AV of PDGF (G9) or VEGF (G10), respectively. An additional $200 \mathrm{~g} /$ rat of ND of VEGF was given to G8 rats on the 7th day after cysteamine administration. Rats were euthanized on the 7th or 14th day after cysteamine and size of duodenal ulcer was measured by morphometric analysis and ulcer area was calculated by the ellipsoid formula. Mucosal scrapings of proximal duodenum were homogenized and tested for VEGF protein using Western blot analysis. The results demonstrated that no one group of rats had significant ulcer healing 7 days after cysteamine compared with controls although the tendency was towards accelerated healing using the PDGF or VEGF vectors. A significant duodenal ulcer healing was noted after 14 days in the groups with $200 \mathrm{~g} / \mathrm{rat}$ of ND of VEGF.i.v. (G8, p=0.0430), $5 \times 10^{8} \mathrm{pfu} / \mathrm{rat}$ of AV of VEGF i.v. (G7, p=0.0025), $5 \times 10^{7}$ pfu/rat i.d. (G5, $\mathrm{p}=0.0198), 5 \times 10^{8} \mathrm{pfu} / \mathrm{rat}$ i.d. $(\mathrm{G} 6, \mathrm{p}=0.0221)$ and $5 \times 10^{8}$ pfu/rat i.v. (G9, p=0.0300) of AV of PDGF, respectively, compared with controls. Western blot analysis showed that the 23 